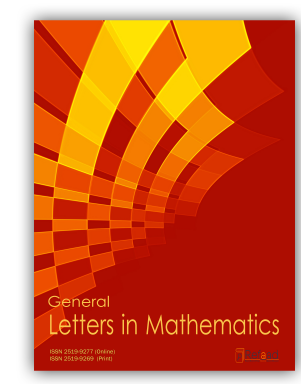

General Letters in Mathematics Vol. 7, No. 1, Sep 2019, pp.1-12

e-ISSN 2519-9277, p-ISSN 2519-9269

Available online at http:// www.refaad.com

https://doi.org/10.31559/glm2019.7.1.1

\title{
Optimal Investment Policy in a Pension Fund System with Return Clause and Multiple Assets under Volatility Risks
}

\author{
Edikan E. Akpanibah ${ }^{*}$, Sylvanus K. Samaila ${ }^{2}$ \\ ${ }^{1,2}$ Department of Mathematics and Statistics,Faculty of Science,Federal University Otuoke, \\ Bayelsa State, Nigeria \\ ${ }^{1}$ edikanakpanibah@gmail.com, ${ }^{2}$ ssylva50@gmail.com
}

\begin{abstract}
The essence of this work is to study the optimal investment policy in a defined contribution pension scheme with return clause of contributions under volatility risks. In our model, the pension fund managers are mandated to return the accumulated contributions of members who die during the accumulation phase to their next of kin. Also, investment in one risk free asset and two risky assets (stock and loan) are considered such that the stock market price is driven by Heston volatility model and the remaining accumulations are distributed among the remaining members. Using mean variance utility function, game theory and variable separation technique, a closed form solution of the optimal investment policy, the optimal fund size and the efficient frontier were obtained. Furthermore, a sensitivity analysis of the effects of some parameters on the optimal investment policies and efficient frontiers were carried out theoretically.
\end{abstract}

Keywords: DC. Pension scheme, extended HJB equation, optimal investment policies, return clause of premium, game theoretic approach, mean variance utility, volatility risk.

2010 MSC No: 91B16, 90C31, 62P05

\section{Introduction}

The defined contribution pension scheme is a retirement scheme whereby a certain percentage of an individual's earning are paid into a retirement savings account (RSA), and the contributions thereafter are invested in a financial market such that the returns from such investments (positive or negative) are paid directly into the member's RSA. In this scheme, the only guaranteed amount in the RSA is the member's monthly contributions but the benefits after retirement is uncertain and depends mostly on investment returns; see [3-5]. For the fact that member's retirement benefits in this scheme depend heavily on the size of fund over the accumulation period, pension fund managers must venture into investment in different assets available in the financial markets with the aim of increasing the accumulated funds; this has lead to the study of optimal portfolio problems and has attract so much attention as it has become so crucial to financial institutions such as insurance companies, commercial bank, pension fund system etc.

Based on this revelations, several authors has engaged in the study of optimal investment policies under different assumptions; some of which include [1], who studied the optimal investment strategy and risk measures of the DC pension plan. [2], investigate the optimal dynamic asset allocation for a defined contribution pension scheme with

\footnotetext{
${ }^{*}$ Corresponding author. Edikan E. Akpanibah ${ }^{1}$ edikanakpanibah@gmail.com
} 
stochastic interest rates and inflation in a continuous-time model. [7], studied stochastic strategies for the optimal investment in a DC pension fund with multiple contributors. [8], studied asset allocation problem under a stochastic interest rate. [9], studied the stochastic optimal control problem often DC pension fund in continuous time. [6], studied optimal investment strategies of the DC pension plan under the stochastic interest rate. From the objective function point of view, there are different types of utility functions used in optimization problem; these include the utility function which shows constant relative risk aversion (CRRA); example power and logarithmic utility functions see [3,10], the utility function which shows constant absolute risk aversion (CARA); example exponential utility, see [13]. The mean-variance utility was first developed by Markowitz to investigate optimal portfolio selection problems see [12], but the challenge is that optimal investment policies under the mean-variance utility are not time consistent, since the mean-variance utility function does not have the iterated expectation condition, hence the Bellman's optimality condition does not hold. However, in many situations time consistency of strategies is a basic requirement for rational decision makers. [14], studied the general theory of Markovian time inconsistent stochastic control problems.

Recently, the study of optimal investment policy with return of contributions clause has taken a centre stage since members of the scheme are faced with mortality risk; these include [15], who investigated optimal investment strategy for a defined contribution pension scheme with the return of premiums clauses in a mean-variance utility function. [16], Investigated equilibrium investment strategy for DC pension plan with default risk and return of premiums clauses under constant elasticity of variance model. [17], Investigated the optimal time-consistent investment strategy for a DC pension with the return of premiums clauses and annuity contracts; they considered investment in two assets, a risk free asset and a risky asset (stock) and assumed the stock market price to follow Heston volatility model. [18], studied Strategic optimal portfolio management for a DC pension scheme with return of premium clauses; in their work, they considered investment in three assets and assume the risky assets are modelled by geometric Brownian motion.

All through these literatures and to the best of our knowledge, no work have been done on optimal investment policy with return of premium clause that considers investment in a cash, stock and loan such that the stock market price is being modelled by Heston volatility model and as such this form the basis of our discussion in this work.

\section{The Model}

Consider a financial market which is complete and frictionless and open continuously over a specific time interval $0 \leq t \leq T, T$ is the retirement age of any given plan member. Also, consider a market which consist of a risk-free asset (cash) and two risky assets; namely (stock) and loan. Suppose $(\Omega, \mathfrak{H}, \mathrm{P})$ is a complete probability space such that $\Omega$ is a real space and $\mathrm{P}$ a probability measure satisfying the condition $0 \leq t \leq T$. $\left\{\dot{B}_{1}(t), \dot{B}_{2}(t), \dot{B}_{3}(t), \dot{B}_{4}(t): t \geq 0\right\}$ are standard Brownian motions. $\mathfrak{H}$ is the filtration and denotes the information generated by the Brownian motions.

Let $\mathfrak{M}_{t}^{1}(t)$ denote the price of the risk free asset, $\mathfrak{M}_{t}^{2}(t)$ the price of the stock which is modelled by the Heston's stochastic volatility model and $\mathfrak{M}_{t}^{3}(t)$ is the price of the loan. Their price models are described as follows:

$$
\begin{gathered}
\frac{d \mathfrak{M}_{t}^{1}(t)}{\mathfrak{M}_{t}^{1}(t)}=\gamma_{1} d t, \mathfrak{M}_{t}^{1}(0)=1 \\
\frac{d \mathfrak{M}_{t}^{2}(t)}{\mathfrak{M}_{t}^{2}(t)}=\left(\gamma_{1}+d_{1} \mathcal{L}(t)\right) d t+\sqrt{L(t)} d \dot{B}_{1} \mathfrak{M}_{t}^{2}(0)=\mathfrak{M}_{0}^{2} \\
\frac{d L_{t}(t)}{L_{t}(t)}=h\left(\frac{\varpi}{L_{t}}-1\right) d t+\eta_{1} \frac{\sqrt{L_{t}}}{L_{t}} d \dot{B_{2}}, \quad L_{t}(0)=l_{0} \\
\frac{d \mathfrak{M}_{t}^{3}(t)}{\mathfrak{M}_{t}^{3}(t)}=\left(\gamma_{1}+d_{2}\right) d t+\eta_{2} d \dot{B_{3}}(t)+\eta_{3} d \dot{B_{4}}(t)
\end{gathered}
$$

Here $\gamma_{1}$ is the predetermined interest rate of the risk free asset and $h, \varpi, d_{1}, d_{2}, \eta_{1}, \eta_{2}, \eta_{3}$ are positive constants and the two Brownian motions $\dot{B}_{1}(t), \dot{B}_{2}(t), \dot{B}_{3}(t)$ and $\dot{B}_{4}(t)$ are such that $d \dot{B}_{1}(t) d \dot{B}_{2}=\rho, d \dot{B}_{1}(t) d \dot{B}_{3}=d \dot{B}_{1}(t) d \dot{B_{4}}=$ $d \dot{B}_{2}(t) d \dot{B}_{3}=d \dot{B}_{2}(t) d \dot{B}_{4}=d \dot{B}_{3}(t) d \dot{B}_{4}=0$ where $\rho$ represent the correlation coefficient of the two Brownian motions and satisfies the condition $-1 \leq \rho \leq 1$.

Let the premium received at a given time be represented as $m$, let $\beta_{0}$ represent the initial age of accumulation phase, $T$, the period of the accumulation phase such that $\beta_{0}+T$ is the end age. The actuarial symbol $\kappa \mathfrak{I}_{\beta_{0}+t}$ is the mortality 
rate from time $t$ to $t+\kappa, b t$ is the premium accumulated at time $\mathrm{t}, t m \kappa \mathfrak{I}_{\beta_{0}+t}$ is the premium returned to the death members.

Let $\ell_{1}, \ell_{2}$, and $\ell_{3}$ represent the proportion of the members pension wealth to be invested in cash, stock and loan respectively such that $\ell_{1}=1-\ell_{2}-\ell_{3}$.

Considering the time interval $[t, t+\kappa]$, the differential form associated with the fund size is given as:

$$
\begin{aligned}
& \mathcal{W}(t+\kappa)=\left(\begin{array}{c}
\mathcal{W}(t)\left(\ell_{1} \frac{\mathfrak{M}_{t+\kappa}^{1}(t)}{\mathfrak{M}_{t}^{1}}+\ell_{2} \frac{\mathfrak{M}_{t+\kappa}^{2}(t)}{\mathfrak{M}_{t}^{2}}+\ell_{3} \frac{\mathfrak{M}_{t+\kappa}^{1}(t)}{\mathfrak{M}_{t}^{1}}\right)+ \\
m \kappa-t m \kappa \mathfrak{T}_{\beta_{0}+t}
\end{array}\right)
\end{aligned}
$$

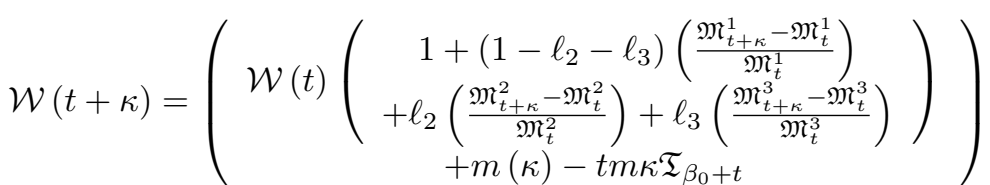

From [11], the conditional death probability $t Q_{y}=1-t P_{y}=1-\exp -\int_{0}^{\kappa} \alpha\left(\beta_{0}+t+s\right) d s$, where $\alpha(t)$ is the force function of the mortality rate at time $t$, and for $\kappa \rightarrow 0$,

$$
\begin{gathered}
\left.\kappa \mathfrak{T}_{\beta_{0}+t}=1-\exp \left(-\int_{0}^{\kappa} \alpha\left(\beta_{0}+t+s\right) d s\right) \approx \alpha \beta_{0}+t\right) \kappa=O(\kappa) \\
\frac{\kappa \mathfrak{T}_{\beta_{0}+t}}{1-\kappa \mathfrak{T}_{\beta_{0}+t}}=\frac{1-\exp \left(-\int_{0}^{\kappa} \alpha\left(\beta_{0}+t+s\right) d s\right)}{\exp \left(-\int_{0}^{\kappa} \alpha\left(\beta_{0}+t+s\right) d s\right)}=\exp \left\{\int_{0}^{\kappa} \alpha\left(\beta_{0}+t+s\right) d s\right\}-1 \approx \alpha\left(\beta_{0}+t\right)=O(\kappa) \\
\kappa \rightarrow 0, \frac{\kappa \mathfrak{T}_{\beta_{0}+t}}{1-\kappa \mathfrak{T}_{\beta_{0}+t}}=\alpha\left(\beta_{0}+t\right) d t, \kappa \mathfrak{T}_{\beta_{0}+t}=\alpha\left(\beta_{0}+t\right) d t m(\kappa) \rightarrow b d t \\
\frac{\mathfrak{M}_{t+\kappa}^{1}-\mathfrak{M}_{t}^{1}}{\mathfrak{M}_{t}^{1}} \rightarrow \frac{d \mathfrak{M}_{t}^{1}(t)}{\mathfrak{M}_{t}^{1}(t)}, \frac{\mathfrak{M}_{t+\kappa}^{2}-\mathfrak{M}_{t}^{2}}{\mathfrak{M}_{t}^{2}} \rightarrow \frac{d \mathfrak{M}_{t}^{2}(t)}{\mathfrak{M}_{t}^{2}(t)}, \frac{\mathfrak{M}_{t+\kappa}^{3}-\mathfrak{M}_{t}^{3}}{\mathfrak{M}_{t}^{3}} \rightarrow \frac{d \mathfrak{M}_{t}^{3}(t)}{\mathfrak{M}_{t}^{3}(t)}
\end{gathered}
$$

Substituting (2.7) into (2.6), we have

$$
\begin{aligned}
\mathcal{W}(t+\kappa) & =\mathcal{W}(t)\left(1+\left(1-\ell_{2}-\ell_{3}\right) \frac{d \mathfrak{M}_{t}^{1}(t)}{\mathfrak{M}_{t}^{1}}+\ell_{2} \frac{d \mathfrak{M}_{t}^{2}(t)}{\mathfrak{M}_{t}^{2}(t)}+\ell_{3} \frac{d \mathfrak{M}_{t}^{3}(t)}{\mathfrak{M}_{t}^{3}(t)}\right) \\
& +m d t-t m \alpha\left(\beta_{0}+t\right) d t \\
d \mathcal{W}(t) & =\left(w(t)\left(\ell_{2} d_{1} L(t)+\ell_{3} d_{2}+\gamma_{1}+m\left(\frac{\beta-\beta_{0}-2 t}{\beta-\beta_{0}-t}\right)\right) d t\right) \\
& +w(t)\left(\ell_{3}\left(\eta_{2} d \dot{B}_{3}(t)+\eta_{3} d \dot{B}_{4}(t)\right)+\ell_{2} \sqrt{\left.(L(t)) d \dot{B}_{1}\right) w(0)}\right. \\
& =w_{0}
\end{aligned}
$$

Where $\beta$ is the maximal age of the life table and $\alpha(t)$ is the force function given by $\alpha(t)=\frac{1}{\beta-t}, \quad 0 \leq t<\beta$

$$
\alpha\left(\beta_{0}+t\right)=\frac{1}{\beta-\beta_{0}-t}
$$

\section{Mean Variance Utility and Extended HJB equation}

Considering the fact that pension accumulations are very volatile, hence there is need for the pension fund managers to formulate the optimal investment problem under the mean-variance criterion as follows:

$$
\sup _{\ell}\left\{E_{t, w, l} \mathcal{W}^{\ell}(T)-\operatorname{Var}_{t, w, l} \mathcal{W}^{\ell}(T)\right\}
$$

Applying the game theoretic method described in [14], the mean-variance control problem in (3.1) is equivalent to the following Markovian time inconsistent stochastic optimal control problem with value function $X(t, w, l)$.

$$
\left\{\begin{array}{c}
Y(t, w, l, \ell)=E_{t, w, l}\left[\mathcal{W}^{\ell}(T)\right]-\frac{\gamma}{2} \operatorname{Var}_{t, w, l}\left[\mathcal{W}^{\ell}(T)\right] \\
Y(t, w, l, \ell)=E_{t, w, l}\left[\mathcal{W}^{\ell}(T)\right]-\frac{\gamma}{2}\left(E_{t, w, l}\left[\mathcal{W}^{\ell}(T)^{2}\right]-\left(E_{t, w, l}\left[\mathcal{W}^{\ell}(T)\right]\right)^{2}\right) \\
X(t, w, l)=\sup _{\ell} Y(t, w, l, \ell)
\end{array}\right.
$$


From [14-15], the optimal portfolio policy $\ell^{*}$ satisfies:

$$
X(t, w, l)=\sup _{\ell} Y\left(t, w, l, \ell^{*}\right)
$$

$\gamma$ is a constant representing risk aversion coefficient of the members.

Let $p^{\ell}(t, w, l)=E_{t, w, l}\left[\mathcal{W}^{\ell}(T)\right], q^{\ell}(t, w, l)=E_{t, w, l}\left[\mathcal{W}^{\ell}(T)^{2}\right]$ then

$$
X(t, w, l) \quad=\sup _{\ell} a\left(t, w, l, p^{\ell}(t, w, l), q^{\ell}(t, w, l)\right)
$$

Where,

$$
a(t, w, l, p, q)=p-\frac{\gamma}{2}\left(q-p^{2}\right)
$$

Theorem 3.1 (verification theorem). If there exist three real functions $\mathcal{A}, \mathcal{B}, \mathcal{C}:[0, T] \times \mathcal{R} \rightarrow \mathcal{R}$ satisfying the following extended Hamilton Jacobi Bellman equations:

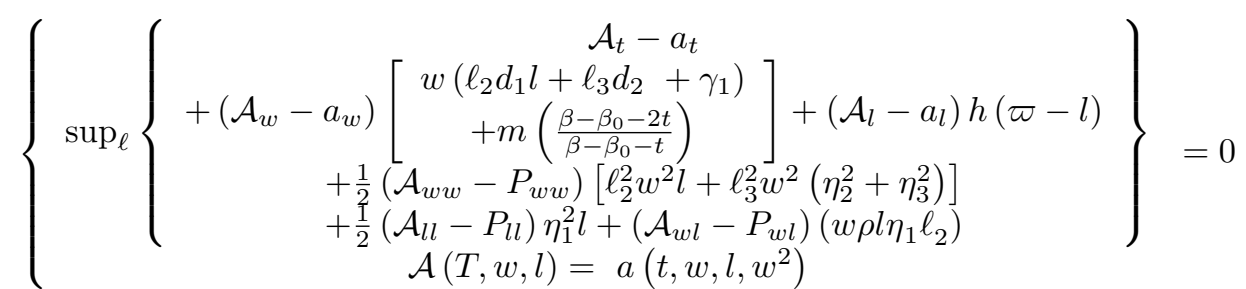

Where,

$$
\begin{aligned}
& \left\{\begin{array}{c}
P_{w w}=a_{w w}+2 a_{w p} p_{w}+2 a_{w q} q_{w}+a_{p p} p_{w}^{2}+2 a_{p q} p_{w} q_{w}+a_{q q} q_{w}^{2}=\gamma \mathcal{B}_{w}^{2} \\
P_{l l}=a_{l l}+2 a_{l p} p_{l}+2 a_{l q} q_{l}+a_{p p} p_{l}^{2}+2 a_{p q} p_{l} q_{l}+a_{q q} q_{l}^{2}=\gamma \mathcal{B}_{l}^{2} \\
P_{w l}=a_{w l}+a_{w p} p_{l}+a_{w q} q_{l}+a_{p l} p_{w}+a_{q l} q_{w}+a_{p p} p_{w} p_{l} \\
+a_{p q} p_{w} q_{l}+a_{p q} p_{l} q_{w}+a_{q q} q_{l} q_{w}=\gamma \mathcal{B}_{w} \mathcal{B}_{l}
\end{array}\right. \\
& \left\{+\mathcal{B}_{w}\left[\begin{array}{c}
w\left(\ell_{2} d_{1} l+\ell_{3} d_{2}+\gamma_{1}\right) \\
+m\left(\frac{\beta-\beta_{0}-2 t}{\beta-\beta_{0}-t}\right) \\
+\frac{1}{2} \mathcal{B}_{w w}\left[\ell_{2}^{2} w^{2} l+\ell_{3}^{2} w^{2}\left(\eta_{2}^{2}+\eta_{3}^{2}\right)\right]+\mathcal{B}_{l} h(\varpi-l) \\
+\frac{1}{2} \mathcal{B}_{l l} \eta_{1}^{2} l+\mathcal{B}_{w l} w \rho l \eta_{1} \ell_{2} \\
\mathcal{B}(T, w, l)=w
\end{array}\right\}=0\right. \\
& \left\{+\mathcal{C}_{w}\left[\begin{array}{c}
w\left(\ell_{2} d_{1} l+\ell_{3} d_{2}+\gamma_{1}\right) \\
+m\left(\frac{\beta-\beta_{0}-2 t}{\beta-\beta_{0}-t}\right) \\
+\frac{1}{2} \mathcal{C}_{w w}\left[\ell_{2}^{2} w^{2} l+\ell_{3}^{2} w^{2}\left(\eta_{2}^{2}+\eta_{3}^{2}\right)\right] \\
+\frac{1}{2} \mathcal{C}_{l l} \eta_{1}^{2} l+\mathcal{C}_{w l} w \rho l \eta_{1} \ell_{2}
\end{array}\right\}=0\right.
\end{aligned}
$$

Then $\mathrm{X}(t, w, l)=\mathcal{A}(t, w, l), p^{\ell^{*}}=\mathcal{B}(t, w, l), q^{\ell^{*}}=\mathcal{C}(t, w, l)$ for the optimal investment policy $\ell^{*}$. See the proof in $[19-21]$

\section{Optimal Investment Policies and Efficient frontier}

In this section, we will attempt to solve equations (3.2), (3.4), and (3.5) to obtain the optimal investment policies and also the efficient frontier.

\section{Proposition 4.1}

The optimal investment policy for the three assets are given as

$$
\ell_{1}^{*}=1-\frac{\left.d_{1} e^{(} \gamma_{1}(t-T)\right)}{\gamma w\left(h+\rho \eta_{1} d_{1}\right)}\left[h+\rho \eta_{1} d_{1} e^{\left(h+\rho \eta_{1} d_{1}\right)(t-T)}\right]-\frac{d_{2} e^{\gamma_{1}(t-T)}}{\gamma w\left(\eta_{2}^{2}+\eta_{3}^{2}\right)}
$$




$$
\begin{gathered}
\ell_{2}{ }^{*}=\frac{d_{1} e^{\gamma_{1}(t-T)}}{\gamma w\left(h+\rho \eta_{1} d_{1}\right)}\left[h+\rho \eta_{1} d_{1} e^{\left(h+\rho \eta_{1} d_{1}\right)(t-T)}\right] \\
\ell_{3}{ }^{*}=\frac{d_{2} e^{\gamma_{1}(t-T)}}{\gamma w\left(\eta_{2}^{2}+\eta_{3}^{2}\right)}
\end{gathered}
$$

Proof

Recall that $a(t, w, l, p, q)=p-\frac{\gamma}{2}\left(q-p^{2}\right)$

$$
a_{t}=a_{w}=a_{l}=a_{w w}=a_{l l}=a_{w l}=a_{p l}=a_{q l}=a_{w p}=a_{w q}=a_{p q}=a_{q q}=0, a_{p}=1+\gamma p, a_{p p}=\gamma, a_{q}=-\frac{\gamma}{2}
$$

Substituting (4.4) into (3.2) and differentiating it with respect to $\ell_{2}$ and $\ell_{3}$, and solving for $\ell_{2}$ and $\ell_{3}$, we have:

$$
\begin{aligned}
& \ell_{2}{ }^{*}=-\left[\frac{d_{1} \mathcal{A}_{w}+\left(\mathcal{A}_{w l}-\gamma \mathcal{B}_{w} \mathcal{B}_{l}\right) \rho \eta_{1}}{w\left(\mathcal{A}_{w w}-\gamma \mathcal{B}_{w}^{2}\right)}\right] \\
& \ell_{3}{ }^{*}=-\left[\frac{d_{2} \mathcal{A}_{w}}{w\left(\mathcal{A}_{w w}-\gamma \mathcal{B}_{w}^{2}\right)\left(\eta_{2}{ }^{2}+\eta_{3}{ }^{2}\right)}\right]
\end{aligned}
$$

Substituting (4.5) and (4.6) into (3.2) and (3.4) we have

$$
\begin{aligned}
& \mathcal{A}_{t}+ \mathcal{A}_{w}\left[\gamma_{1} w+m\left(\frac{\beta-\beta_{0}-2 t}{\beta-\beta_{0}-t}\right)\right]+\mathcal{A}_{l} h(\varpi-l)+\frac{1}{2}\left(\mathcal{A}_{l l}-\gamma \mathcal{B}_{l}^{2}\right) \eta_{1}^{2} l=0 \\
&- \frac{1}{2} \frac{\mathcal{A}_{w}^{2} d_{2}^{2}}{\left(\mathcal{A}_{w w}-\gamma \mathcal{B}_{w}^{2}\right)\left(\eta_{2}{ }^{2}+\eta_{3}{ }^{2}\right)}-\frac{l}{2}\left(\frac{\left(d_{1} \mathcal{A}_{w}+\left(\mathcal{A}_{w l}-\gamma \mathcal{B}_{w} \mathcal{B}_{l}\right) \rho \eta_{1}\right)^{2}}{\left(\mathcal{A}_{w w}-\gamma \mathcal{B}_{w}^{2}\right)}\right) \\
& \mathcal{B}_{t}+\mathcal{B}_{w}\left[\gamma_{1} w+m\left(\frac{\beta-\beta_{0}-2 t}{\beta-\beta_{0}-t}\right)\right]+\mathcal{B}_{l} h(\varpi-l)++\frac{1}{2} \mathcal{B}_{l l} \eta_{1}^{2} l \\
& \\
&-\left(l k_{1} \mathcal{B}_{w}+\rho \sigma_{1} l \mathcal{B}_{w l}\right)\left(\frac{d_{1} \mathcal{A}_{w}+\left(\mathcal{A}_{w l}-\gamma \mathcal{B}_{w} \mathcal{B}_{l}\right) \rho \eta_{1}}{\left(\mathcal{A}_{w w}-\gamma \mathcal{B}_{w}^{2}\right)}\right) \\
&-\frac{\mathcal{B}_{w} \mathcal{A}_{w} d_{2}^{2}}{\left(\mathcal{A}_{w w}-\gamma \mathcal{B}_{w}^{2}\right)\left(\eta_{2}{ }^{2}+\eta_{3}{ }^{2}\right)}+\frac{1}{2} l \mathcal{B}_{w w}\left(\frac{d_{1} \mathcal{A}_{w}+\left(\mathcal{A}_{w l}-\gamma \mathcal{B}_{w} \mathcal{B}_{l}\right) \rho \eta_{1}}{\left(\mathcal{A}_{w w}-\gamma \mathcal{B}_{w}^{2}\right)}\right)^{2}
\end{aligned}
$$

Next, we assume a solution for $\mathcal{A}(t, w, l)$ and $\mathcal{B}(t, w, l)$ as follows:

$$
\left\{\begin{array}{c}
\mathcal{A}(t, z, l)=w f_{1}(t)+\frac{l}{\gamma} f_{2}(t) \frac{1}{\gamma} f_{3}(t), \quad f_{1}(T)=1, f_{2}(T)=0 \quad f_{3}(T)=0 \\
\mathcal{B}(t, z, l)=w g_{1}(t)+\frac{l}{\gamma} g_{2}(t)+\frac{1}{\gamma} g_{3}(t), \quad g_{1}(T)=1, g_{2}(T)=0 \quad g_{3}(T)=0 \\
\mathcal{A}_{t}=w \frac{d f_{1}(t)}{d t}+\frac{l}{\gamma} \frac{d f_{2}(t)}{d t}+\frac{1}{\gamma} \frac{d f_{3}(t)}{d t}, \mathcal{A}_{w}=f_{1}(t), \mathcal{A}_{w w}=0, \mathcal{A}_{l}=\frac{f_{2}(t)}{\gamma}, \mathcal{A}_{l l}=0 \\
\mathcal{B}_{t}=w \frac{d g_{1}(t)}{d t}+\frac{l}{\gamma} \frac{d g_{2}(t)}{d t}+\frac{1}{\gamma} \frac{d g_{3}(t)}{d t}, \mathcal{B}_{w}=g_{1}(t), \mathcal{B}_{w w}=0, \mathcal{B}_{l}=\frac{g_{2}(t) l}{\gamma}, \mathcal{B}_{l l}=0
\end{array}\right.
$$

Substituting (4.9) into (4.7) and (4.8), we have

$$
\begin{aligned}
& \left\{\begin{array}{c}
\frac{d f_{1}(t)}{d t}+\gamma_{1} f_{1}(t)=0 \\
\frac{d f_{2}(t)}{d t}-h f_{2}-\frac{\sigma_{1}^{2} g_{2}{ }^{2}}{2}+\frac{k_{1}^{2} f_{1}{ }^{2}}{2 g_{1}{ }^{2}}+\frac{\rho^{2} \sigma_{1}^{2} g_{2}{ }^{2}}{2}-\frac{\rho \sigma_{1} k_{1} f_{1} g_{2}}{g_{1}}=0 \\
\frac{d f_{3}(t)}{d t}+h \varpi f_{2}+f_{1} m \gamma\left(\frac{\beta-\beta_{0}-2 t}{\beta-\beta_{0}-t}\right)+\frac{k_{2}^{2} f_{1}^{2}}{2\left(\eta_{2}{ }^{2}+\eta_{3}{ }^{2}\right) g_{1}{ }^{2}}=0
\end{array}\right. \\
& \left\{\begin{array}{c}
\frac{d g_{1}(t)}{d t}+\gamma_{1} g_{1}(t)=0 \\
\frac{d g_{2}(t)}{d t}-h g_{2}+\frac{k_{1}^{2} f_{1}}{g_{1}}-\frac{\rho \sigma_{1} k_{1} f_{1} g_{2}}{g_{1}}=0 \\
\frac{d g_{3}(t)}{d t}+h \varpi g_{2}+g_{1} m \gamma\left(\frac{\beta-\beta_{0}-2 t}{\beta-\beta_{0}-t}\right)+\frac{f_{1} g_{1} k_{2}^{2}}{\left(\eta_{2}{ }^{2}+\eta_{3}{ }^{2}\right) g_{1}{ }^{2}}=0
\end{array}\right.
\end{aligned}
$$

Solving (4.10) and (4.11), we have

$$
\begin{aligned}
& f_{1}(t)=e^{\gamma_{1}(T-t)} \\
& f_{2}(t)=\frac{\rho \eta_{1} d_{1}^{3}}{h+\rho \eta_{1} d_{1}}\left\{\begin{array}{c}
\frac{1}{h}\left(e^{h(t-T)}-1\right) \\
+\frac{1}{\rho \eta_{1} d_{1}}\left(e^{h(t-T)}-e^{\left(h+\rho \eta_{1} d_{1}\right)(t-T)}\right)
\end{array}\right\}+\frac{d_{1}^{2}}{2 h}\left(1-e^{h(t-T)}\right) \\
& +\frac{\eta_{1}^{2} d_{1}^{3}\left(\rho^{2}-1\right)}{2\left(h+\rho \eta_{1} d_{1}\right)^{2}}\left\{\begin{array}{c}
\frac{1}{h}\left(e^{h(t-T)}-1\right) \\
+\frac{2 e^{h(t-T)}}{\rho \eta_{1} d_{1}}\left(1-e^{\rho \eta_{1} d_{1}(t-T)}\right)+ \\
\frac{e^{h(t-T)}}{h+2 \rho \eta_{1} d_{1}}\left(e^{\left(h+\rho \eta_{1} d_{1}\right)(t-T)}-1\right)
\end{array}\right\}
\end{aligned}
$$




$$
\begin{aligned}
& f_{3}(t)=-h \varpi \int_{t}^{T} f_{2}(\tau) d \tau+m \gamma \int_{t}^{T} \frac{\tau e^{\gamma_{1}(T-\tau)}}{\beta-\beta_{0}-\tau} d \tau+\frac{m \gamma}{r_{1}}\left(e^{\gamma_{1}(t-T)}-1\right)+\frac{d_{2}^{2}}{2\left(\eta_{2}^{2}+\eta_{3}^{2}\right)}(T-t) \\
& g_{1}(t)=e^{\gamma_{1}(T-t)} \\
& g_{2}(t)=\frac{d_{1}^{2}}{h+\rho \eta_{1} d_{1}}\left(1-e^{\left(h+\rho \eta_{1} d_{1}\right)(t-T)}\right) \\
& g_{3}(t)=-h \varpi \int_{t}^{T} g_{2}(\tau) d \tau+m \gamma \int_{t}^{T} \frac{\tau e^{\gamma_{1}(T-\tau)}}{\beta-\beta_{0}-\tau} d \tau+\frac{m \gamma}{r_{1}}\left(e^{\gamma_{1}(t-T)}-1\right)+\frac{d_{2}^{2}}{2\left(\eta_{2}^{2}+\eta_{3}^{2}\right)}(T-t) \\
& \mathcal{A}(t, w, l)=\left(\begin{array}{c}
w e^{\gamma_{1}(T-t)} \\
\frac{\rho \eta_{1} d_{1}^{3}}{h+\rho \eta_{1} d_{1}}\left\{\begin{array}{c}
\frac{1}{h}\left(e^{h(t-T)}-1\right) \\
+\frac{1}{\rho \eta_{1} d_{1}}\left(e^{h(t-T)}-e^{\left(h+\rho \eta_{1} d_{1}\right)(t-T)}\right) \\
+\frac{d_{1}^{2}}{2 h}\left(1-e^{h(t-T)}\right)
\end{array}\right\} \\
+\frac{\eta_{1}^{2} d_{1}^{3}\left(\rho^{2}-1\right)}{2\left(h+\rho \eta_{1} d_{1}\right)^{2}}\left\{\begin{array}{c}
\frac{1}{h}\left(e^{h(t-T)}-1\right) \\
+\frac{2 e^{h(t-T)}}{\rho \sigma_{1} d_{1}}\left(1-e^{\rho \sigma_{1} d_{1}(t-T)}\right)+ \\
\frac{e^{h(t-T)}}{h+2 \rho \eta_{1} d_{1}}\left(e^{\left(h+\rho \eta_{1} d_{1}\right)(t-T)}-1\right)
\end{array}\right\} \\
+\frac{1}{\gamma}\left[\begin{array}{c}
T \varpi \int_{t}^{T} f_{2}(\tau) d \tau \\
+m \gamma \int_{t}^{T} \frac{\tau e^{\gamma_{1}(T-\tau)}}{\beta-\beta_{0}-\tau} d \tau \\
+\frac{m \gamma}{\gamma_{1}}\left(e^{\gamma_{1}(t-T)}-1\right) \\
+\frac{d_{2}^{2}}{2\left(\eta_{2}{ }^{2}+\eta_{3}{ }^{2}\right)}(T-t)
\end{array}\right]
\end{array}\right\} \\
& \left.\mathcal{B}(t, w, l)=\left(\begin{array}{c}
w e^{\gamma_{1}(T-t)} \\
+\frac{l}{\gamma}\left\{\frac{d_{1}^{2}}{h+\rho \eta_{1} d_{1}}\left(1-e^{\left(h+\rho \eta_{1} d_{1}\right)(t-T)}\right)\right\} \\
+h \varpi \int_{t}^{T} g_{2}(\tau) d \tau \\
+m \gamma \int_{t}^{T} \frac{\tau e^{\gamma_{1}(T-\tau)}}{\beta-\beta_{0}-\tau} d \tau \\
+\frac{m \gamma}{\gamma_{1}}\left(e^{\gamma_{1}(t-T)}-1\right) \\
+\frac{d_{2}^{2}}{2\left(\eta_{2}{ }^{2}+\eta_{3}{ }^{2}\right)}(T-t)
\end{array}\right\}\right) \\
& \mathcal{A}_{w}=f_{1}(t), \mathcal{B}_{w}=g_{1}(t), \mathcal{A}_{w w}=0 \text {, and } \mathcal{B}_{l}=g_{2}(t)
\end{aligned}
$$

Substituting (4.14) into (4.5) and (4.6), we have

$$
\begin{gathered}
\ell_{1}{ }^{*}=1-\ell_{2}{ }^{*}-\ell_{3}{ }^{*}=1-\frac{d_{1} e^{\gamma_{1}(t-T)}}{\gamma w\left(h+\rho \eta_{1} d_{1}\right)}\left[h+\rho \eta_{1} d_{1} e^{\left(h+\rho \eta_{1} d_{1}\right)(t-T)}\right]-\frac{d_{2} e^{\gamma_{1}(t-T)}}{\gamma w\left(\eta_{2}{ }^{2}+\eta_{3}{ }^{2}\right)} \\
\ell_{2}{ }^{*}=\frac{d_{1} e^{\gamma_{1}(t-T)}}{\gamma w\left(h+\rho \eta_{1} d_{1}\right)}\left[h+\rho \eta_{1} d_{1} e^{\left(h+\rho \eta_{1} d_{1}\right)(t-T)}\right] \\
\ell_{3}{ }^{*}=\frac{d_{2} e^{\gamma_{1}(t-T)}}{\gamma w\left(\eta_{2}{ }^{2}+\eta_{3}{ }^{2}\right)}
\end{gathered}
$$

\section{Proposition 4.2}

The efficient frontier of the pension members is given by

$$
E_{t, w, l}\left[\mathcal{W}^{\ell^{*}}(T)\right]=w e^{\gamma_{1}(T-t)}+\sqrt{\frac{V a r_{t, w, l}\left[\mathcal{W}^{\ell^{*}}(T)\right]}{I(t)}}\left\{\begin{array}{c}
\frac{l d_{1}^{2}}{h+\rho \eta_{1} d_{1}}\left(1-e^{\left(h+\rho \eta_{1} d_{1}\right)(t-T)}\right) \\
-h \varpi \int_{t}^{T} g_{2}(\tau) d \tau \\
+m \gamma \int_{t}^{T} \frac{\tau e^{\gamma_{1}(T-\tau)}}{\beta-\beta_{0}-\tau} d \tau \\
+\frac{m \gamma}{\gamma_{1}}\left(e^{\gamma_{1}(t-T)}-1\right) \\
+\frac{d_{2}^{2}}{2\left(\eta_{2}{ }^{2}+\eta_{3}{ }^{2}\right)}(T-t)
\end{array}\right\}
$$

Proof

$$
\operatorname{Var}_{t, w, l}\left[\mathcal{W}^{\ell^{*}}(T)\right]=E_{t, w, l}\left[\mathcal{W}^{\ell}(T)^{2}\right]-\left(E_{t, w, l}\left[\mathcal{W}^{\ell}(T)\right]\right)^{2}=\frac{2}{\gamma}(\mathcal{B}(t, w, l)-\mathcal{A}(t, w, l))
$$


Substituting (4.12) and (4.13) into the above equation, we have

$$
\begin{aligned}
& \operatorname{Var}_{t, w, l}\left[\mathcal{W}^{\ell^{*}}(T)\right]=\frac{1}{\gamma^{2}}\left(2 l\left\{\begin{array}{c}
\left(\frac{d_{1}^{2}}{h+\rho \eta_{1} d_{1}}+\frac{\eta_{1}^{2} d_{1}^{3}\left(\rho^{2}-1\right)}{2\left(h+\rho \eta_{1} d_{1}\right)^{2}}+\frac{e^{h(t-T)}}{h+2 \rho \eta_{1} d_{1}}\right)\left(1-e^{\left(h+\rho \eta_{1} d_{1}\right)(t-T)}\right) \\
+\frac{d_{1}^{2}}{2 h}\left(1-e^{h(t-T)}\right) \\
-\left[\begin{array}{c}
\frac{1}{h}\left(e^{h(t-T)}-1\right) \\
\frac{\rho \eta_{1} d_{1}^{3}}{h+\rho \eta_{1} d_{1}}\left\{\begin{array}{c}
1 \\
+\frac{1}{\rho \eta_{1} d_{1}}\left(e^{h(t-T)}-e^{\left(h+\rho \eta_{1} d_{1}\right)(t-T)}\right) \\
+\frac{\sigma_{1}^{2} d_{1}^{3}\left(\rho^{2}-1\right)}{2\left(h+\rho \eta_{1} d_{1}\right)^{2}}\left\{\begin{array}{c}
\frac{1}{h}\left(e^{h(t-T)}-1\right) \\
+\frac{2 e^{h(t-T)}}{\rho \eta_{1} d_{1}}\left(1-e^{\rho \eta_{1} d_{1}(t-T)}\right)
\end{array}\right\} \\
+2 h \varpi \int_{t}^{T}\left(f_{2}(\tau)-g_{2}(\tau)\right) d \tau
\end{array}\right\}
\end{array}\right\}
\end{array}\right\}\right. \\
& \operatorname{Var}_{t, w, l}\left[\mathcal{W}^{\ell^{*}}(T)\right]=\frac{1}{\gamma^{2}} I(t)
\end{aligned}
$$

Where

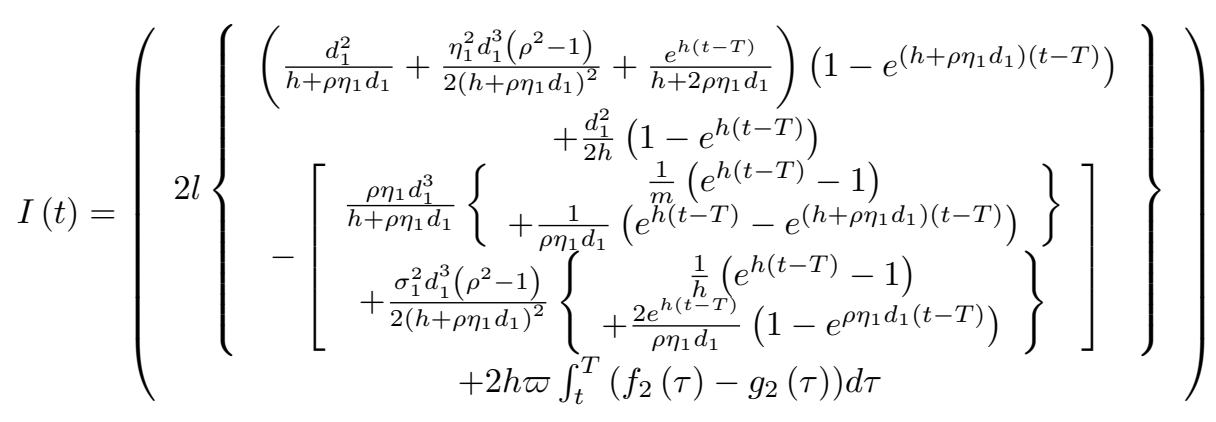

$$
\begin{aligned}
& \frac{1}{\gamma}=\frac{\sqrt{\operatorname{Var}_{t, w, l}\left[\mathcal{W}^{\ell^{*}}(T)\right]}}{\sqrt{I(t)]}} \\
& E_{t, w, l}\left[\mathcal{W}^{\ell^{*}}(T)\right]=\mathcal{B}(t, w, l) \\
& E_{t, w, l}\left[\mathcal{W}^{\ell^{*}}(T)\right]=w e^{\gamma_{1}(T-t)}+\frac{1}{\gamma}\left\{\begin{array}{c}
\frac{l d_{1}^{2}}{h+\rho \eta_{1} d_{1}}\left(1-e^{\left(h+\rho \eta_{1} d_{1}\right)(t-T)}\right) \\
-h \varpi \int_{t}^{T} g_{2}(\tau) d \tau \\
+m \gamma \int_{t}^{T} \frac{\tau e^{\gamma_{1}(T-\tau)}}{\beta-\beta_{0}-\tau} d \tau \\
+\frac{m \gamma}{\gamma_{1}}\left(e^{\gamma_{1}(t-T)}-1\right) \\
+\frac{d_{2}^{2}}{2\left(\eta_{2}{ }^{2}+\eta_{3}{ }^{2}\right)}(T-t)
\end{array}\right\}
\end{aligned}
$$

Substituting (4.15) i (4.16), we have

$$
E_{t, w, l}\left[\mathcal{W}^{\ell^{*}}(T)\right]=w e^{\gamma_{1}(T-t)}+\sqrt{\frac{V a r_{t, w, l}\left[\mathcal{W}^{\ell^{*}}(T)\right]}{I(t)}}\left\{\begin{array}{c}
\frac{l d_{1}^{2}}{h+\rho \eta_{1} d_{1}}\left(1-e^{\left(h+\rho \eta_{1} d_{1}\right)(t-T)}\right) \\
-h \varpi \int_{t}^{T} g_{2}(\tau) d \tau \\
+m \gamma \int_{t}^{T} \frac{\tau e^{\gamma_{1}(T-\tau)}}{\beta-\beta_{0}-\tau} d \tau \\
+\frac{m \gamma}{?_{1}}\left(e^{\gamma_{1}(t-T)}-1\right) \\
+\frac{d_{2}^{2}}{2\left(\eta_{2}{ }^{2}+\eta_{3}{ }^{2}\right)}(T-t)
\end{array}\right\}
$$


Proposition 4.3 The optimal fund size $\mathcal{W}^{\ell^{*}}(t)$ corresponding to the optimal allocation strategy $\ell^{*}$ is given as

$$
\begin{aligned}
\mathcal{W}(t) & =\frac{\bar{w} h d_{1}}{\gamma}+\left(\left(\frac{t}{h}-\frac{1}{h^{2}}\right) e^{h t}+\frac{1}{h^{2}}\right)\left(1-\frac{\rho \eta_{1} d_{1}}{h+\rho \eta_{1} d_{1}}\right) e^{(} \gamma_{1}(t-T) \\
& \left.+e^{h t}\left(\frac{t}{2 h+\rho \eta_{1} d_{1}}-\frac{1}{\left(2 h+\rho \eta_{1} d_{1}\right)^{2}}\right) e^{(} \gamma_{1}+h+\rho \eta_{1} d_{1}\right)((t-T) \\
& +\left(\frac{e^{(}-\left(h+\rho \eta_{1} d_{1}\right) T}{\left(2 h+\rho \eta_{1} d_{1}\right)^{2}} e^{(} \gamma_{1}(t-T)\right) \\
& +\frac{d_{1}^{2}}{h \gamma}\left[\exp \left(\gamma_{1}(t-T)\right)\left(1-\frac{\rho \eta_{1} d_{1}}{h\left(h+\rho \eta_{1} d_{1}\right)}\right)\right. \\
& +\frac{1}{2 h+\rho \eta_{1} d_{1}} \exp \left(\gamma_{1}+h+\rho \eta_{1} d_{1}(t-T)\right] \exp (h t-1) \\
& +\frac{t d_{2}^{2} \exp \left(\gamma_{1}(t-T)\right)}{\gamma\left(\eta_{2}^{2}+\eta_{3}^{2}\right)}+w_{0} \exp \left(\gamma_{1} t\right) \\
& +\frac{m}{h}\left(\exp \left(\gamma_{1} t-1\right)\right)-h \gamma \exp \left(\gamma_{1} t\right) \int_{t}^{T}\left(\frac{\tau e^{-}\left(\gamma_{1} t\right)}{\beta-\beta_{0}-\tau}\right) d \tau
\end{aligned}
$$

Proof From equation (2.9) we have

$$
d \mathcal{W}(t)=\left(\begin{array}{c}
\left(\begin{array}{c}
\mathcal{W}(t)\left(\ell_{2} d_{1} L(t)+\ell_{3} d_{2}+\gamma_{1}\right) \\
+m\left(\frac{\beta-\beta_{0}-2 t}{\beta-\beta_{0}-t}\right)
\end{array}\right. \\
\left.\mathcal{W}(t)\left(\ell_{3}\left(\eta_{2} d_{3}(t)+\eta_{3} d \dot{B}_{4}(t)\right)+\ell_{2} \sqrt{(L}(t)\right) d \dot{B}_{1}\right)
\end{array}\right) d t+\mathcal{W}(0)=w_{0}
$$

Where $L(t)=(h \varpi t+1) e^{m t}$

Solving the linear differential equation, we have the result above

\section{Sensitivity Analysis}

Proposition 5.1 Suppose $w>0, \gamma>0, \gamma_{1}>0, t \in[0, T] h>0, d_{1}>0, \eta_{1}>0, \gamma_{1}>0$ and $\rho \in[-1,1]$ then $(i)$

$\frac{\partial \ell_{2}^{*}}{\partial w}<0 \quad(i i) \frac{\partial \ell_{2}^{*}}{\partial \gamma}<0 \quad$ (iii) $\frac{\partial \ell_{2}^{*}}{\partial \gamma_{1}}<0 \quad$ (iv) $\frac{\partial \ell_{2}^{*}}{\partial t}>0$
Proof

(i) Recall that $\ell_{2}{ }^{*}=\frac{d_{1} e^{\gamma_{1}(t-T)}}{\gamma w\left(h+\rho \eta_{1} d_{1}\right)}\left[h+\rho \eta_{1} d_{1} e^{\left(h+\rho \eta_{1} d_{1}\right)(t-T)}\right]$

$$
\frac{\partial \ell_{2}{ }^{*}}{\partial w}=-\frac{d_{1} e^{\gamma_{1}(t-T)}}{\gamma w^{2}\left(h+\rho \eta_{1} d_{1}\right)}\left[h+\rho \eta_{1} d_{1} e^{\left(h+\rho \eta_{1} d_{1}\right)(t-T)}\right]
$$

For $\rho=0$

$$
\frac{\left[h+\rho \eta_{1} d_{1} e^{\left(h+\rho \eta_{1} d_{1}\right)(t-T)}\right]}{h+\rho \eta_{1} d_{1}}=\frac{h}{h}=1>0
$$

For $\rho<0$,

$\frac{\left[h+\rho \eta_{1} d_{1} e^{\left(h+\rho \eta_{1} d_{1}\right)(t-T)}\right]}{h+\rho \eta_{1} d_{1}}>0$ since either $\rho \eta_{1} d_{1} e^{\left(h+\rho \eta_{1} d_{1}\right)(t-T)}<0$ when $\rho \eta_{1} d_{1}<0$ and $\rho \eta_{1} d_{1} e^{\left(h+\rho \eta_{1} d_{1}\right)(t-T)}>0$ when $\rho \eta_{1} d_{1}>0$

For $\rho>0$,

$$
\frac{\left[h+\rho \eta_{1} d_{1} e^{\left(h+\rho \eta_{1} d_{1}\right)(t-T)}\right]}{h+\rho \eta_{1} d_{1}}>0
$$

Therefore for all $\rho \in[-1,1], \frac{\left[h+\rho \eta_{1} d_{1} e^{\left(h+\rho \eta_{1} d_{1}\right)(t-T)}\right]}{h+\rho \eta_{1} d_{1}}>0$

Since $\frac{d_{1} e^{\gamma_{1}(t-T)}}{\gamma w^{2}}>0$ and $\frac{\left[h+\rho \eta_{1} d_{1} e^{\left(h+\rho \eta_{1} d_{1}\right)(t-T)}\right]}{h+\rho \eta_{1} d_{1}}>0$. Hence

$$
\frac{\partial \ell_{2}{ }^{*}}{\partial w}=-\frac{d_{1} e^{\gamma_{1}(t-T)}}{\gamma w^{2}\left(h+\rho \eta_{1} d_{1}\right)}\left[h+\rho \eta_{1} d_{1} e^{\left(h+\rho \eta_{1} d_{1}\right)(t-T)}\right]<0
$$


(ii) $\frac{\partial \ell_{2}{ }^{*}}{\partial \gamma}=-\frac{d_{1} e^{\gamma_{1}(t-T)}}{\gamma^{2} w\left(h+\rho \eta_{1} d_{1}\right)}\left[h+\rho \eta_{1} d_{1} e^{\left(h+\rho \eta_{1} d_{1}\right)(t-T)}\right]$

Since $\frac{d_{1} e^{\gamma_{1}(t-T)}}{\gamma^{2} w}>0$ and $\frac{\left[h+\rho \eta_{1} d_{1} e^{\left(h+\rho \eta_{1} d_{1}\right)(t-T)}\right]}{h+\rho \eta_{1} d_{1}}>0$. Hence

$$
\frac{\partial \ell_{2}^{*}}{\partial \gamma}<0
$$

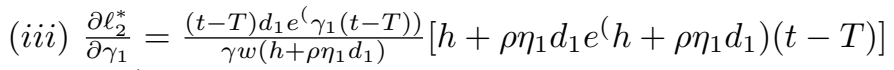

Since $\frac{\left.d_{1} e^{(} \gamma_{1}(t-T)\right)}{\gamma w}>0, \frac{h+\rho \eta_{1} d_{1} \exp \left(h+\rho \eta_{1} d_{1}\right)(t-T)}{h+\rho \eta_{1} d_{1}}>0$ and $(t-T)<0$. Hence $\frac{\partial \ell_{2}^{*}}{\partial \gamma_{1}}<0$

(iv) $\ell_{2}^{*}=\frac{d_{1} \exp \left(\gamma_{1}(t-T)\right)}{\gamma w\left(h+\rho \eta_{1} d_{1}\right)}\left[h+\rho \eta_{1} d_{1} e^{\left(h+\rho \eta_{1} d_{1}\right)(t-T)}\right]=\frac{h d_{1} e^{\gamma_{1}(t-T)}}{\gamma w\left(h+\rho \eta_{1} d_{1}\right)}+\frac{\rho \eta_{1} d_{1}^{2} e^{\left(\gamma_{1}+h+\rho \eta_{1} d_{1}\right)(t-T)}}{\gamma w\left(h+\rho \eta_{1} d_{1}\right)}$

$$
\frac{\partial \ell_{2}^{*}}{\partial t}=\frac{\gamma_{1} h d_{1} e^{\gamma_{1}(t-T)}}{\gamma w\left(h+\rho \eta_{1} d_{1}\right)}+\frac{\left(\gamma_{1}+h+\rho \eta_{1} d_{1}\right) \rho \eta_{1} d_{1}^{2} e^{\left(\gamma_{1}+h+\rho \eta_{1} d_{1}\right)(t-T)}}{\gamma w\left(h+\rho \eta_{1} d_{1}\right)}
$$

Since $\gamma_{1} h d_{1} e^{(} \gamma_{1}(t-T)>0$ and $\left.\left(\gamma_{1}+h+\rho \eta_{1} d_{1}\right) \rho \eta_{1} d_{1}^{2} e^{(} \gamma_{1}+h+\rho \eta_{1} d_{1}\right)(t-T)>0$, then $\frac{\partial \ell_{2}^{*}}{\partial t}>0$

\section{Proposition 5.2}

Suppose $w>0, \gamma>0, d_{2}>0, \eta_{2}>0, \eta_{3}>0, \gamma_{1}>0, t \in[0, T]$ then (i) $\frac{\partial \ell_{3}{ }^{*}}{\partial w}<0$ (ii) $\frac{\partial \ell_{3}{ }^{*}}{\partial \gamma}<0$ (iii) $\frac{\partial \ell_{3}{ }^{*}}{\partial \gamma_{1}}<0$ (iv) $\frac{\partial \ell_{3}^{*}}{\partial t}>0$

Proof

(i) Recall that $\ell_{3}{ }^{*}=\frac{d_{2} e^{\gamma_{1}(t-T)}}{\gamma w\left(\eta_{2}{ }^{2}+\eta_{3}{ }^{2}\right)}$

$$
\frac{\partial \ell_{3}{ }^{*}}{\partial w}=-\frac{d_{2} e^{\gamma_{1}(t-T)}}{\gamma w^{2}\left(\eta_{2}{ }^{2}+\eta_{3}^{2}\right)}
$$

Since $e^{\gamma_{1}(t-T)}>0$ and $\frac{d_{2}}{\gamma w^{2}\left(\eta_{2}{ }^{2}+\eta_{3}{ }^{2}\right)}>0$ then

$$
\frac{\partial \ell_{3}^{*}}{\partial w}=-\frac{d_{2} e^{\gamma_{1}(t-T)}}{\gamma w^{2}\left(\eta_{2}^{2}+\eta_{3}^{2}\right)}<0
$$

(ii) $\frac{\partial \ell_{3}{ }^{*}}{\partial \gamma}=-\frac{d_{2} e^{\gamma_{1}(t-T)}}{\gamma^{2} w\left(\eta_{2}{ }^{2}+\eta_{3}{ }^{2}\right)}$

Since $e^{\gamma_{1}(t-T)}>0$ and $\frac{d_{2}}{\gamma^{2} w\left(\eta_{2}{ }^{2}+\eta_{3}{ }^{2}\right)}>0$. Hence

$$
\frac{\partial \ell_{3}^{*}}{\partial \gamma}=-e^{\gamma_{1}(t-T)} \cdot \frac{d_{2}}{\gamma^{2} w\left(\eta_{2}^{2}+\eta_{3}^{2}\right)}<0
$$

(iii) $\frac{\partial \ell_{3}{ }^{*}}{\partial \gamma_{1}}=(t-T) \frac{d_{2} e^{\gamma_{1}(t-T)}}{\gamma w\left(\eta_{2}{ }^{2}+\eta_{3}{ }^{2}\right)}$

Since $\frac{d_{2} e^{\gamma_{1}(t-T)}}{\gamma w\left(\eta_{2}{ }^{2}+\eta_{3}{ }^{2}\right)}>0$ and $t<T$, implies that $t-T<0$. Hence $\frac{\partial \ell_{3}^{*}}{\partial \gamma_{1}}<0$ (iv) $\ell_{3}^{*}=\frac{d_{2} e^{\gamma_{1}(t-T)}}{\gamma w\left(\eta_{2}{ }^{2}+\eta_{3}{ }^{2}\right)}$

$$
\frac{\partial \ell_{3}^{*}}{\partial t}=\frac{\gamma_{1} d_{2} e^{\gamma_{1}(t-T)}}{\gamma w\left(\eta_{2}^{2}+\eta_{3}^{2}\right)}
$$

Since $e^{\gamma_{1}(t-T)}>0$ and $\frac{\gamma_{1} d_{2}}{\gamma w\left(\eta_{2}{ }^{2}+\eta_{3}{ }^{2}\right)}>0$. Hence

$$
\frac{\partial \ell_{3}^{*}}{\partial t}>0
$$

Proposition 5.3 Suppose $h>0, w>0, \gamma>0, \gamma_{1}>0, t \in[0, T], d_{1}>0, d_{2}>0, \eta_{1}>0, \eta_{2}>0, \eta_{3}>0$, and $\rho \in[-1,1]$ then (i) $\frac{\partial \ell_{1}{ }^{*}}{\partial w}>0$ (ii) $\frac{\partial \ell_{1}{ }^{*}}{\partial \gamma}>0$ (iii) $\frac{\partial \ell_{1}{ }^{*}}{\partial \gamma_{1}}>0$ (iv) $\frac{\partial \ell_{1}{ }^{*}}{\partial t}<0$

$$
\ell_{1}^{*}=1-\frac{d_{1} e^{\gamma_{1}(t-T)}}{\gamma w\left(h+\rho \eta_{1} d_{1}\right)}\left[h+\rho \eta_{1} d_{1} e^{\left(h+\rho \eta_{1} d_{1}\right)(t-T)}\right]-\frac{d_{2} e^{\gamma_{1}(t-T)}}{\gamma w\left(\eta_{2}^{2}+\eta_{3}^{2}\right)}
$$


(i) $\ell_{1}^{*}=1-\left(\ell_{2}^{*}-\ell_{3}^{*}\right)$

$$
\frac{\partial \ell_{1}{ }^{*}}{\partial w}=-\frac{\partial \ell_{2}{ }^{*}}{\partial w}-\frac{\partial \ell_{3}{ }^{*}}{\partial w}=-\left(\frac{\partial \ell_{2}{ }^{*}}{\partial w}+\frac{\partial \ell_{3}{ }^{*}}{\partial w}\right)
$$

But $\frac{\partial \ell_{2}{ }^{*}}{\partial w}<0$ and $\frac{\partial \ell_{3}{ }^{*}}{\partial w}<0$, therefore $\frac{\partial \ell_{2}{ }^{*}}{\partial w}+\frac{\partial \ell_{3}{ }^{*}}{\partial w}<0$. hence

$$
\frac{\partial \ell_{1}{ }^{*}}{\partial w}=-\left(\frac{\partial \ell_{2}{ }^{*}}{\partial w}+\frac{\partial \ell_{3}{ }^{*}}{\partial w}\right)>0
$$

(ii) $\frac{\partial \ell_{1}{ }^{*}}{\partial \gamma}=-\frac{\partial \ell_{2}{ }^{*}}{\partial \gamma}-\frac{\partial \ell_{3}{ }^{*}}{\partial \gamma}=-\left(\frac{\partial \ell_{2}{ }^{*}}{\partial \gamma}+\frac{\partial \ell_{3}{ }^{*}}{\partial \gamma}\right)$

Since $\frac{\partial \ell_{2}{ }^{*}}{\partial \gamma}<0$ and $\frac{\partial \ell_{3}{ }^{*}}{\partial \gamma}<0$, such that $\frac{\partial \ell_{2}{ }^{*}}{\partial \gamma}+\frac{\partial \ell_{3}{ }^{*}}{\partial \gamma}<0$. Therefore

$$
\frac{\partial \ell_{1}^{*}}{\partial \gamma}>0
$$

(iii) $\frac{\partial \ell_{1}{ }^{*}}{\partial \gamma_{1}}=-\frac{\partial \ell_{2}{ }^{*}}{\partial \gamma_{1}}-\frac{\partial \ell_{3}{ }^{*}}{\partial \gamma_{1}}=-\left(\frac{\partial \ell_{2}{ }^{*}}{\partial \gamma_{1}}+\frac{\partial \ell_{3}^{*}}{\partial \gamma_{1}}\right)$

Since $\frac{\partial \ell_{2}^{*}}{\partial \gamma_{1}}<0$ and $\frac{\partial \ell_{3}^{*}}{\partial \gamma_{1}}<0$, such that $\frac{\partial \ell_{2}^{*}}{\partial \gamma_{1}}+\frac{\partial \ell_{3}^{*}}{\partial \gamma_{1}}<0$. Therefore

$$
\frac{\partial \ell_{1}^{*}}{\partial \gamma_{1}}>0
$$

(iii) $\frac{\partial \ell_{1}^{*}}{\partial t}=-\frac{\partial \ell^{*}}{\partial t}-\frac{\partial \ell_{3}^{*}}{\partial t}=-\left(\frac{\partial \ell_{2} *}{\partial t}+\frac{\partial \ell_{3}^{*}}{\partial t}\right)$

Since $\frac{\partial \ell_{2}{ }^{*}}{\partial t}>0$ and $\frac{\partial \ell_{3}{ }^{*}}{\partial t}>0$, such that $\frac{\partial \ell_{2}{ }^{*}}{\partial t}+\frac{\partial \ell_{3}{ }^{*}}{\partial t}>0$. Therefore

$$
\frac{\partial \ell_{1}^{*}}{\partial t}<0
$$

\section{Discussion}

Based on our observation, proposition 5.1 and 5.2 shows that the optimal portfolio policies of the two risky assets are inversely proportional to the risk aversion coefficient, initial fund size and the predetermined interest rate and directly proportional to time. Also, proposition 5.3 shows that the optimal portfolio policy of the risk free asset is directly proportional to the risk aversion coefficient, initial fund size and the predetermined interest rate and inversely proportional to time.

The implications of the above propositions is that members who are scared to take risk should invest more in risk free asset while members who love taking risk can invest in more in risky asset to increase their expectations. Secondly, if the initial fund size at the time of investment is high, members may decide to reduce the amount of risk to be taken, thereby reducing the proportion of their funds to be invested in risky assets. Thirdly, when the risk free investment offers high interest rate, members will be attracted to such investment since human being naturally does not like much risk but prefers good returns, they will prefer to invest more in risk free asset at the expense of the risky assets. Finally, we observed that as retirement age of the remaining members draw closer, there will be anxiety in the mind of these members on what their final accumulations will be like considering the effect of the return clause which has taken some good amount out of the system, these members will like to increase their investments in risky assets in order to boost their investment return since DC pension members' benefit depend on the investment returns.

From equation (4.15), we observed that the risk aversion coefficient is inversely proportional to the variance; this shows that members of the scheme with high risk aversion coefficient will prefer to invest more in risk free asset and invest less in risky assets and vice versa. Also, equation (4.17), we observed that the expectation is directly dependent on the variance; this means that when members take more risk, their expectation from such investment will be higher compared to the members who invest less in the risky asset.

\section{Conclusion}

We investigated optimal investment policy in a pension fund system (DC) with return of premium clauses under Heston's Volatility model using mean-variance utility function. Investments in cash, equity and loan were considered 
to help increase the accumulated funds of the remaining members to meet their retirement needs. We established the extended Hamilton Jacobi Bellman equations using and obtained the optimal investment policies for the three assets and also the efficient frontier using game theoretic approach and variable separable method. A theoretical analysis of the effects of some parameters on the optimal investment policies was given. We conclude that the optimal investment policies of the risky assets decrease with the initial fund size, predetermined interest rate, risk aversion coefficient and increases as retirement age approaches.

\section{References}

1. S. Haberman, E. Vigna, 2002. Optimal investment strategies and risk measures in defined contribution pension schemes. Insurance: Mathematics and Economics 31, 35-69. https://doi.org/10.1016/s0167-6687(02)00128-2

2. N. Han, M. Hung (2012). Optimal asset allocation for the DC pension plans under inflation. Insurance: Mathematics and Economics 51: 172-181. https://doi.org/10.1016/j.insmatheco.2012.03.003

3. A. J. G. Cairns, D. Blake, K. Dowd. Stochastic lifestyling: optimal dynamic asset allocation for defined contribution pension plans, Journal of Economic Dynamics and Control 30(5)(2006) 843-877. https://doi.org/10.1016/j.jedc.2005.03.009

4. Y. Wang, S. Fan and H. Chang. (2018). DC Pension Plan with the Return of Premium Clauses under Inflation Risk and Volatility Risk J. Sys. Sci. and Math. Scis. 38(4): 423-437.

5. B. O Osu, E. E Akpanibah., K.N.C Njoku, (2017). On the Effect of Stochastic Extra Contribution on Optimal Investment Strategies for Stochastic Salary under the Affine Interest Rate Model in a DC Pension Fund.General Letters in Mathematics, vol. 2(3), 138-149.

https://doi.org/10.31559/glm2016.2.3.5

6. J. F. Boulier, S. Huang, G. Taillard G. Optimal management under stochastic interest rates: the case of a protected defined contribution pension fund, Insurance 28(2)(2001), 173-189. https://doi.org/10.1016/s0167-6687(00)00073-1

7. E. E. Akpanibah, S. K. Samaila. (2017)Stochastic strategies for optimal investment in a defined contribution (DC) pension fund, International Journal of Applied Science and Mathematical Theory, 3(3)(2017), 48-55.

8. J. Gao. Stochastic optimal control of DC pension funds, Insurance, 42(3)(2008), 1159-1164. https://doi.org/10.1016/j.insmatheco.2008.03.004

9. L. He, Z. Liang, 2013. Optimal dynamic asset allocation Strategy for ELA scheme of DC pension plan during the distribution phase. Insurance: Mathematics \& Economics 52(2), 404-410 https://doi.org/10.1016/j.insmatheco.2013.02.005

10. C. Zhang, X Rong. (2013). Optimal investment strategies for DC pension with astochastic salary under affine interest rate model. Hindawi Publishing Corporation, vol 2013 https://doi.org/10.1155/2013/297875

11. Kohler, P.-H., Kohler, I., 2000. Frailty modeling for adult and old age mortality: the application of a modified De Moivre Hazard function to sex differentials in mortality. Demographic Research 3. https://doi.org/10.4054/demres.2000.3.8

12. Markowitz, H.M. (1952). Portfolio selection. Journal of Finance 7: 77-91.

13. E. E. Akpanibah and B. O. Osu (2017). Portfolio Strategy for an Investor with Stochastic Premium under Exponential Utility via Legendre Transform and Dual Theory. International Journal of Advances in Mathematics. Volume 2017, Number 6, Pages 27-35.

14. T. Björk, A. Murgoci. A general theory of Markovian time inconsistent stochastic control problems. Working Paper. Stockholm School of Economics. http : //ssrn.com/abstract = 1694759. (2009). 
15. L. He, Z. Liang. The optimal investment strategy for the DC plan with the return of premiums clauses in a mean-variance framework, Insurance, 53(2013), 643-649. https://doi.org/10.1016/j.insmatheco.2013.09.002

16. D Li, X. Rong, H. Zhao, B. Yi. Equilibrium investment strategy for DC pension plan with default risk and return of premiums clauses under CEV model, Insurance 72(2017), 6-20 https://doi.org/10.1016/j.insmatheco.2016.10.007

17. D. Sheng, X. Rong. Optimal time consistent investment strategy for a DC pension with the return of premiums clauses and annuity contracts, Hindawi Publishing Corporation vol (2014) 1-13. https://doi.org/10.1155/2014/862694

18. E. E. Akpanibah, B. O. Osu, B. I. Oruh and C. N. Obi (2019). Strategic Optimal Portfolio Management for a Dc Pension Scheme with Return Of Premium Clauses. Transactions of the Nigerian Association of Mathematical Physics, 8(1): 121-130.

19. L. He, Z. Liang. Optimal financing and dividend control of the insurance company with fixed and proportional transaction costs. Insurance: Mathematics \& Economics 44(2009), 88-94. https://doi.org/10.1016/j.insmatheco.2008.10.001

20. Z. Liang, J. Huang. Optimal dividend and investing control of an insurance company with higher solvency constraints. Insurance: Mathematics \& Economics 49(2011), 501-511. https://doi.org/10.1016/j.insmatheco.2011.08.008

21. Y. Zeng, Z. Li. Optimal time consistent investment and reinsurance policies for mean-variance insurers. Insurance: Mathematics \& Economics 49(2011), 145-154.

https://doi.org/10.1016/j.insmatheco.2011.01.001 